\title{
Embriopatía por isotretinoína: Un daño evitable
}

\author{
Mónica Troncoso Sch ${ }^{1}$, Carla Rojas $\mathrm{H}^{1}$, Eduardo Bravo $\mathrm{C}^{2}$. \\ Isotretinoin embryophaty. \\ Report of one case
}

Retinoic acid is a widely used drug in the treatment of cystic acne. It has teratogenic effects that depend on the gestational period in which it is used. We report a seven months old female whose mother was exposed to retinoic acid in both pregestational and gestational periods. She had a retardation of psychomotor development and a brain MRI showed frontal atrophy and a malformation of the posterior fossa. We discuss the mechanisms of the teratogenic effects of retinoic acid (Rev Méd Chile 2008; 136: 763-6).

(Key words: Abnormalities, drug-induced; Embryonic and fetal development; Tretinoin)

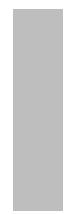

Recibido el 30 de marzo, 2007. Aceptado el 4 de septiembre, 2007.

${ }^{1}$ Servicio de Neuropsiquiatría Infantil, Hospital Clínico San Borja Arriarán y Departamento de Pediatría, Campus Centro, Facultad de Medicina, Universidad de Chile. ${ }^{2}$ Servicio de Neurorradiología Diagnóstica y Terapéutica, Instituto de Neurocirugía "Dr. Asenjo". Departamento de Ciencias Neurológicas Oriente, Facultad de Medicina, Universidad de Chile. Santiago de Chile.

$\mathrm{P}$ osterior a la introducción de la isotretinoína (13 cis ácido retinoico), en 1980, para el tratamiento del acné quístico severo, se reportaron varios casos de embriopatía en hijos de madres expuestas dentro de los 15 días posterior a la concepción ${ }^{1}$.

Cerca de $35 \%$ de los niños expuestos en el período gestacional nacen con malformaciones craneofaciales (microtia o anotia, estenosis del canal auditivo externo, parálisis facial, micrognatia, puente nasal bajo, hipertelorismo, paladar ojival); tímicas (ectopia, hipoplasia); cardiológicas (defecto septo ventricular, malformaciones conotroncales, hipoplasia ventrículo derecho) y del sistema nervioso central (SNC) (hidrocefalia, microcefalia, hipoplasia cerebelar, retardo mental,

Correspondencia a: Dra. Carla Rojas H. Santa Rosa 1234, Santiago, Chile. Fonos: (56-2) 544.65.68 - (56-2) 544.65.79. Fax: 5555343. E mail: carlarojash@yahoo.com espina bífida, agenesia vermis cerebeloso, heterotopías leptomeníngeas, anomalías tracto corticoespinal y holoprosencefalia ${ }^{2-4}$.

\section{OBJETIVo}

Describimos el caso clínico de una lactante con secuelas neurológicas secundarias a exposición materna periconcepcional a ácido retinoico.

\section{CASO CLÍNICO}

Lactante de 7 meses, primera hija de padre sano y madre en tratamiento por acné quístico con ácido retinoico, $10 \mathrm{mg}$ al día, durante 3 meses previos al embarazo, indicado por dermatólogo, quien le informó los riesgos de su uso.

En un control en el segundo mes de embarazo se suspendió el ácido retinoico. Al tercer mes 
presentó síntomas de parto prematuro. La hija nació por parto vaginal, de término, adecuada para edad gestacional, sin asfixia. Presentó dificultades en succión e ictericia que requirió fototerapia, sin otras complicaciones.

Evolucionó con retraso del desarrollo psicomotor (RDSM) global. A los 6 meses se hospitalizó por un cuadro diarreico, objetivándose síndrome hipotónico, RDSM global y anisocoria. Se realizó tomografía axial computarizada cerebral que mostró aumento del espacio subaracnoideo, signos de atrofia frontal y probable malformación de la fosa posterior. En nuestro Servicio se encontró una circunferencia craneana normal, episodios de hipernea, poco contacto ocular, apraxia ocular, anisocoria con midriasis del ojo derecho y fondo de ojo con retina pigmentada sin acúmulos anormales, hipotonía axial con aumento del tono muscular distal con predominio en las extremidades inferiores, sin clonus y plantar extensor bilateral. Destacaba, además, una historia de constipación crónica.

Los exámenes bioquímicos en sangre y orina, acilcarnitinas, electrofisiología (electroencefalograma, potenciales evocados visuales y auditivos), cariograma, manometría del tubo digestivo, resultaron normales. La evaluación neuroftalmológica mostró papilas normales con anisocoria del ojo derecho mayor que izquierdo, sin reflejo fotomotor bilateral, sugerente de una lesión mesencefálica dorsal.

La resonancia nuclear magnética cerebral mostró agenesia del vermis cerebeloso (Figuras 1 y 2).

\section{Discusión}

Las malformaciones congénitas de la fosa posterior y las manifestaciones neurológicas e intestinales de esta paciente podrían ser explicadas por la exposición materna preconcepcional y postconcepcional a ácido retinoico administrado por vía oral. Esto ha sido reportado en estudios animales y casos clínicos en humanos ${ }^{1-4}$.

La vitamina A y sus derivados, llamados retinoides (naturales o sintéticos), son necesarios para procesos vitales: visión, reproducción, crecimiento, diferenciación celular, función inmune y embriogénesis ${ }^{5}$. El exceso o déficit dietario de vitamina A, resulta teratogénico durante el perio- do gestacional. El déficit previo o durante la gestación, puede producir malformaciones oculares, urogenitales, diafragmáticas y cardiopulmonares $^{6}$. El exceso, puede producir toxicidad en el SNC, hígado, huesos y piel ${ }^{7}$.

El ácido retinoico se administra por vía oral, transdérmica o parenteral. Estudios en animales

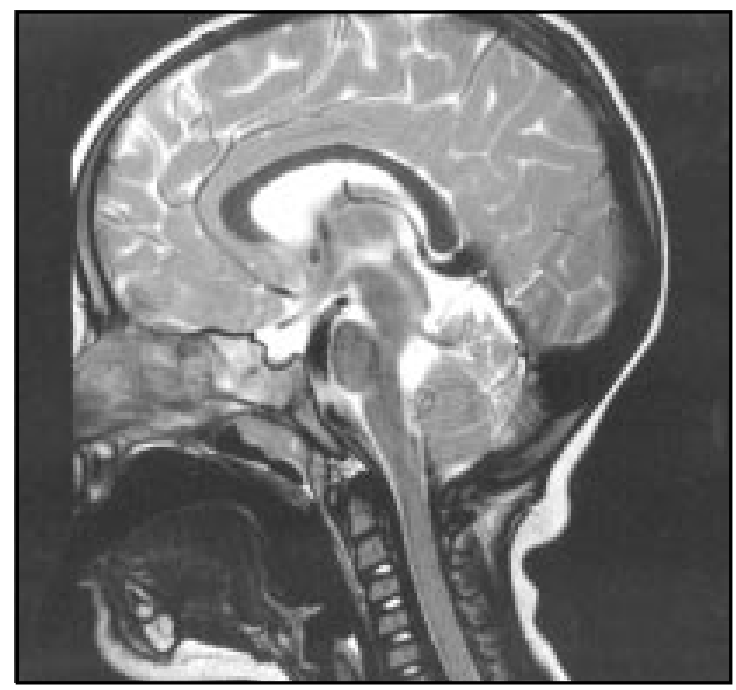

Figura 1. Resonancia magnética. Secuencia sagital ponderada en T2. Se observa agenesia del vermis cerebeloso.

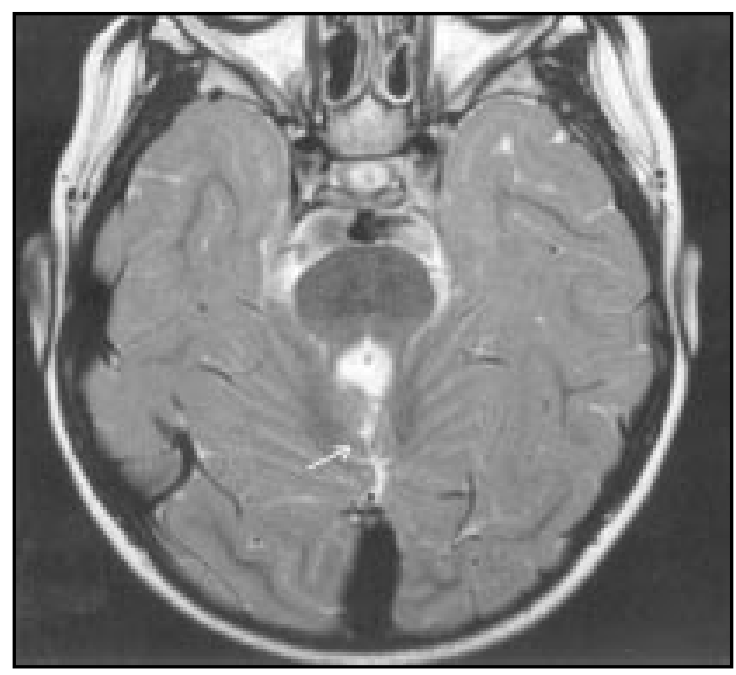

Figura 2. Resonancia magnética. Secuencia axial ponderada en T2. Se observa agenesia del vermis cerebeloso. 
de experimentación señalan que la vía transdérmica es la que tiene la menor biodisponibilidad, con dosis por cada aplicación de $0,15 \mathrm{mg} / \mathrm{kg}$, la que se encuentra muy por debajo de las dosis teratogénicas en humanos expuestos ${ }^{8}$. Sin embargo, existe un caso de malformaciones congénitas en el hijo de una madre usuaria, durante los 5 primeros meses de gestación, de un preparado tópico con

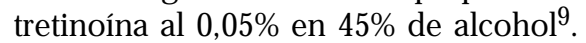

En nuestra paciente se observó que el consumo oral de este medicamento en dosis estándar, provocó malformaciones de la fosa posterior cerebral durante su gestación.

La dosis de isotretinoína necesaria para gene$\mathrm{rar}$ teratogenicidad en humanos ( $1 \mathrm{mg} / \mathrm{kg} /$ día) es menor a la dosis mínima necesaria teratogénica en animales, explicado por diferente toxicocinética, transferencia placentaria y metabolismo ${ }^{10}$.

En el caso de los primates, éstos son particularmente sensibles a la forma cis de la isotretinoína, debido a su lenta eliminación, baja detoxificación, falta de unión a proteína ligantes de retinoides con posibilidad de acceder fácilmente al núcleo y continua isomerización hacia la forma trans del ácido retinoico, el cual presenta la propiedad de distribuirse ampliamente a través de la placenta, lo que explicaría el potencial teratogénico de la isotretinoína ${ }^{10}$.

Los períodos de mayor susceptibilidad a la teratogénesis ocurren en la fase de organogénesis ( $3^{\mathrm{a}}$ y 8 $^{\mathrm{a}}$ semanas) considerando que cada malformación tiene un periodo crítico propio ${ }^{11}$.

Los retinoides inducen una perturbación de las células de la cresta neural y las células no diferenciadas de la población celular neural. La baja expresión de genes homeobox dlx inducida por retinoides en células de la cresta neural, genera malformaciones craneofaciales, no así en aquellas que no expresan dichos genes ${ }^{12}$.

En modelos experimentales en ratas, con la exposición a retinoides en periodos específicos de la embriogénesis (día 9,5-10,5) se encontraron anomalías malformativas en núcleos pontinos y olivar inferior, por falla en la migración neurofílica.

La formación de células compactas distribuidas desde el labio rómbico anterior al puente ventral, es reemplazada por libre distribución en la médula anterior ${ }^{13}$.

Estos datos podrían explicar los hallazgos encontrados en nuestra paciente, tales como malformación estructural de fosa posterior y anomalías funcionales manifestadas como alteración en el reflejo pupilar, movimientos oculares y pausas respiratorias más hipernea.

Otros estudios muestran que la exposición de embriones de ratas a retinoides, en los días 10, 12 ó 17, induce alteraciones histopatológicas en más de $50 \%$ en el SNC, arcos branquiales y sus derivados (excesivo repliegue y elevación del piso del tubo neural, bolsa de Rathke persistente, ganglio trigeminal pequeño, divertículo neural o defectos ópticos y óticos). La exposición en el día 17 de gestación provocó malformaciones en la implantación, reabsorción y, en fetos aparentemente normales, alteraciones en la osificación. Esto confirma que el desarrollo de estructuras neurales y derivados de la cresta neural se altera con la exposición a retinoides previo a la formación de la placa neural, sugiriendo que proteínas reguladas por el retinol se encuentran presentes tempranamente en el desarrollo embrionario de los vertebrados ${ }^{14}$.

En el periodo fetal, se altera la producción de células del SNC, generando desórdenes funcionales. En modelos animales genera disminución de neuroblastos, espasticidad, temblor e hiperactividad ${ }^{12}$.

La teratogenicidad induce unos 200 genes con un fenotipo similar. Las alteraciones objetivadas incluyen mutaciones, no disyunción cromosómica, interferencia mitótica, alteración del funcionamiento del ácido nucleico, déficit de sustratos o precursores energéticos, inhibición enzimática, alteración de la membrana celular y desbalance osmolar. Estos mecanismos producen muerte celular, reducción de la biosíntesis, falla en interacción celular y disrupción.

El mecanismo teratogénico del retinol es semejante al inducido por el etanol o al déficit de folatos, entre otros.

En el síndrome alcohol fetal, el etanol induciría alteraciones en la síntesis endógena de retinol generando un déficit de éste, especialmente en el cerebelo postnatal, región particularmente vulnerable. En modelos experimentales, los astrocitos tienen aumento de la capacidad de sintetizar retinol en presencia de etanol mediante una enzima deshidrogenada, lo que explicaría la relación entre ambos teratógenos ${ }^{15}$.

Aunque existen dos receptores específicos para retinol (RAR y RXR), a través de modelos 
experimentales animales se precisó que la teratogenicidad es mediada por RAR, al observar la disminución de malformaciones como espina bífida, atresia anal, micrognatia, con el uso de antagonistas de RAR. La administración de antagonistas y su efecto es dependiente de la edad gestacional a la cual se realiza el procedimiento ${ }^{16}$.

En conclusión, los reportes de casos clínicos de pacientes gestantes expuestas a isotretinoína, asociado a los hallazgos en estudios experimentales en animales, son la base de la implementación por parte de la Food and Drug Administration (FDA) de los Estados Unidos de Norteaménica, desde 1988, de programas de prevención de embarazo en mujeres en edad fértil usuarias de retinol en Estados Unidos de Norteamérica,

\section{REFERENCIAS}

1. Boyie R. Effects of certain prenatal drugs on the fetus and newborn. Pediatr Rev 2002; 23: 17-23.

2. Fernhoff P, Lammer E. Craniofacial features of isotretinoin embriophathy. J Pediatr 1984; 105: 583-97.

3. Hansen L, Pearl GS. Isotretinoin teratogenicity. Case report with neuropathologic finding. Acta Neuropathol 1985; 65: 335-7.

4. Cohen MM, Shiota K. Teratogenesis of Holoprosencephaly. J Med Genet 2002; 109: 1-15.

5. Cowns M, MaO G. Teratology of Retinoids. Ann Rev Pharmacol 1999; 39: 399-430.

6. MorRISs-Kay GM, Sokolova N. Embryonic development and pattern formation. FASEB J 1996; 10: 961-8.

7. Teelmann K. Retinoids: Toxicology and Teratogenicity to date. Pharmacol Ther 1989; 40: 29-34.

8. NAU H. Embriotoxicity and teratogenicity of topical retinoid acid. Skin Pharmacol 1993; suppl 1: 35-44.

9. Lipson AH, Cowns F, WeBsTer WS. Multiple congenital defects associated with maternal use of topical tretinoin. Lancet 1993; 341: 1352-53.

10. NAU H. Teratogenicity of isotretinoin revisited: species variation and the rol of all trans-retinoic acid. J Am Acad Dermatol 2001; 45: S183-187.

11. SHENEFELT RE. Morphogenesis of malformations in hamsters caused by retinoic acid: relation to dose and stage of treatment. Teratology 1972; 5: 103-18.

12. Langman J, Welch GW. Excess vitamin $A$ and development of the cerebral cortex. J Comp Neurol 1967; 131: 15-26. dando instrucción del niesgo teratogénico y exigiendo tener un test de embarazo negativo más un ciclo menstrual activo previo al inicio de la terapia ${ }^{16}$. Desde el año 2000 es obligatorio realizar encuestas de control a toda mujer en edad fértil usuaria de retinol ${ }^{17-19}$.

Sin embargo, aún no se ha conseguido obtener una tasa de embarazo igual a cero en las usuarias de retinol, probablemente por baja comprensión de los riesgos y falta de rigurosidad en la aplicación de métodos anticonceptivos ${ }^{20}$.

Es indispensable, por lo tanto, que los profesionales de la salud den a conocer el riesgo teratogénico del ácido retinoico durante el embarazo para otorgar la información suficiente a toda mujer en edad fértil potencialmente usuaria de este medicamento.

13. Yamamoto $M$, Zhang J, Smith D, Hayakawa $Y$, Mc CEFFERY P. A critical period for retinoid acid teratogenesis and loss of neurophilic migration of pontine nuclei neurons. Mech Dev 2003; 120: 701-9.

14. Pauken CM, La Borde JB, Bolon B. Retinoic acid acts during peri-implantational development to alter axial and brain formation. Anat Embryol 1999; 200: 645-55.

15. Mc Caffery P, Koul O, Smith D, Napol JL, Chen N, ULman MD. Ethanol increases retinoic acid production in cerebellar astrocytes and in cerebellum. Brain Res Dev 2004; 153: 233-41.

16. Elmazar M M, Ruhl R, Reichert U, Shroot B, NaU $\mathrm{H}$. RAR alpha mediated teratogenicity in mice is potentiated by RXR agonist and reduced by RAR antagonist: dissection of retinoid receptor-induced pathways. Toxicol Appl Pharmacol 1997; 146: 21-8.

17. Buck M. Isotretinoin improving patient education and reducing risk. Pediatr Pharmacother 2001; v7 n7.

18. Holmes SC, BankowsKa U, Macje RM. The prescription of isotretinoin to women is very precaution taken? Br J Dermatol 1998; 138: 450-5.

19. AтACACKovic G, Koren G. Fetal exposure to oral isotretinoin: failure to comply with the pregnancy prevention program. CMAJ 1999; 160: 1719-20.

20. Bensouda L, Jonvile AP, Mouret E, Elefant E, Dнешот $\mathrm{H}$, Delmas $\mathrm{C}$ et al. Isotretinoin: compliance with recommendations in childbearing women. Ann Dermatol Venereol 2005; 132: 415-23. 